\title{
Novel insights by 4D Flow imaging on aortic flow physiology after valve-sparing root replacement with or without neosinuses ${ }^{\dagger}$
}

\author{
Nicola Galea ${ }^{\mathrm{a}, *}$, Filippo Piatti ${ }^{\mathrm{b}}$, Francesco Sturla ${ }^{\mathrm{b}, \mathrm{c}}$, Jonathan W. Weinsaft ${ }^{\mathrm{d}}$, Christopher Lau ${ }^{\mathrm{e}}$, Ilaria Chirichilli ${ }^{\mathrm{e}}$, \\ lacopo Carbone ${ }^{a}$, Emiliano Votta ${ }^{b}$, Carlo Catalano ${ }^{a}$, Ruggero De Paulis ${ }^{f}$, Leonard N. Girardi ${ }^{e}$, Alberto Redaelli ${ }^{b}$ \\ and Mario Gaudino ${ }^{\mathrm{e}}$, the Cornell International Consortium for Aortic Surgery (CICAS) ${ }^{\mathbf{e}}$
}

\author{
a Department of Radiological, Oncological and Pathological Sciences, Sapienza University of Rome, Rome, Italy \\ b Department of Electronics, Information and Bioengineering, Politecnico di Milano, Milan, Italy \\ c 3D and Computer Simulation Laboratory, IRCCS Policlinico San Donato, San Donato Milanese, Italy \\ d Departments of Medicine (Cardiology), Weill Cornell Medicine, New York, NY, USA \\ e Department of Cardiothoracic Surgery, Weill Cornell Medicine, New York, NY, USA \\ f Department of Cardiac Surgery, European Hospital, Rome, Italy \\ * Corresponding author. Department of Radiological, Oncological and Pathological Sciences, "Sapienza" University of Rome, viale Regina Elena 324,00161 Rome, \\ Italy. Tel: +39-6-3282231647; e-mail: nicola.galea@gmail.com (N. Galea).
}

Received 7 September 2017; received in revised form 30 November 2017; accepted 9 December 2017

\begin{abstract}
OBJECTIVES: This study was undertaken to evaluate the flow dynamics in the aortic root after valve-sparing root replacement with and without neosinuses of Valsalva reconstruction, by exploiting the capability of 4D Flow imaging to measure in vivo blood velocity fields and 3D geometric flow patterns.
\end{abstract}

METHODS: Ten patients who underwent valve-sparing root replacement utilizing grafts with neosinuses or straight tube grafts ( 5 cases each) were evaluated by 4D Flow imaging at a mean of 46.5 months after surgery. We used in-house processing tools to quantify relevant bulk flow variables (flow rate, stroke volume, peak velocity and mean velocity), wall shear stresses and the amount of flow rotation characterizing the region enclosed by the graft and the aortic valve leaflets.

RESULTS: Despite bulk flows with similar peak velocities, flow rates and stroke volumes $(P=0.31-1.00)$, the neosinuses graft was associated with a lower mean velocity $(P<0.03)$ and magnitude of wall shear stress along the axial direction of the vessel wall $(P<0.05)$ at the proximal root level but remained comparable along the circumferential direction $(P=0.22-1.0)$ to the straight tube graft. Flow rotation was evidently and systematically higher in the neosinuses grafts, characterized by streamline rotations higher than $270^{\circ}$, nearly triple that of tubular grafts $(10.3 \div 14.0 \%$ of all aortic streamline vs $2.2 \div 5.7 \%, P=0.008)$.

CONCLUSIONS: Recreation of the sinuses of Valsalva during valve-sparing root replacement is associated with significantly lower wall shear stress and organized vortical flows at the level of the sinus that are not evident using the straight tube graft. These findings need confirmation in larger studies and could have important implications in terms of aortic valve durability.

Keywords: Aortic root replacement $\cdot$ Valve-sparing aortic root replacement $\cdot$ Neosinuses graft $\cdot$ Vortex $\cdot$ Sinuses of Valsalva $\cdot$ 4D Flow imaging

\section{INTRODUCTION}

Valve-sparing aortic root replacement (VSRR) is an attractive surgical option for the treatment of aortic root aneurysms with preserved cusps [1]. In the traditional David VSRR procedure, the aortic root is replaced with a straight cylindrical tube graft in which the native valve is reimplanted [2]. Among the many technical evolutions proposed in VSRR surgery, new grafts designed with the recreation of the sinuses of Valsalva (SV) have gained

tPresented at the 31st Annual Meeting of the European Association for CardioThoracic Surgery, Vienna, Austria, 7-10 October 2017. increasing interest as they may reproduce native aortic flow physiology $[3,4]$.

The importance of vortical flow generated in the SV in modulating the leaflet movements and regulating progressive and low stress closure of the aortic valve has been extensively demonstrated in vivo and in experimental models [5-8]. However, the clinical advantages offered by a surgical approach preserving the physiological aortic flow dynamics and its impact on valve mechanics still remain a matter of debate [3, 9-12].

$3 \mathrm{D}$ phase-contrast magnetic resonance (4D Flow) imaging has emerged as a powerful non-invasive technique to elucidate vascular 
flow characteristics due to its capability to analyse in vivo blood velocity fields and 3D flow patterns [13, 14].

Volumetric 4D Flow imaging not only allows the 3D geometric evaluation of helical and vortical blood flow formations [13] but also enables the assessment of derived flow parameters, such as the wall shear stresses (WSS) that measure the tangential forces exerted by flowing blood acting on the aortic wall $[15,16]$.

The aim of this study was to compare the fluid dynamic effects of neosinuses reconstruction in patients who had undergone VSRR by using 4D Flow imaging to explore the intra-vessel fluid dynamics and the corresponding mechanical loads acting on the aortic wall, as measured via magnetic resonance imaging-derived computational analysis.

\section{MATERIALS AND METHODS}

\section{Study population}

Ten patients who underwent aortic VSRR for the treatment of aortic root aneurysms without associated aortic valve disease were enrolled. All patients had technically satisfactory surgical results (none/trivial aortic regurgitation). Surgery was performed at 2 sites using 2 different surgical techniques (see below): the NY Presbyterian Hospital/Weill Cornell Medicine (WCMC, $n=5$ ) and the European Hospital/Rome $(E H R, n=5)$. Patients from each site were age- and gender matched; all were followed up postoperatively for at least 6 months. Patients were excluded based on technically inadequate surgical repair ( $\geq$ mild aortic insufficiency), genetically mediated aortopathies (e.g. bicuspid aortic valve and Marfan syndrome) or contraindication to magnetic resonance imaging.

The institutional review board or ethics committee at each participating centre approved the study; written informed consent was obtained from all patients.

\section{Surgical technique}

Surgical technique was indicated by valvular and aortic pathological criteria according to the established best practice, European Society of Cardiology (ESC)/European Association for CardioThoracic Surgery (EACTS) [17] and American College of Cardiology/American Heart Association (ACC/AHA) [18] guidelines. At the WCMC, the classical David I technique was used with reimplantation of the aortic annulus into a straight Dacron tube without SV (Macquet Corp., Fairfield, NJ, USA), whereas at the EHR, a graft with recreation of neo-SV (graft with neo-SV) was used (Vascutek $^{\circledR}$ Gelweave Valsalva ${ }^{\text {TM }}$ Grafts, Terumo, Inchinnan, Scotland). Details of the surgical techniques used in the 2 centres have been previously published $[4,10,19]$.

\section{D Flow acquisitions}

All magnetic resonance (MR) examinations were performed on identical 3.0-T magnetic resonance imaging units (Discovery MR750, GE Healthcare) at the Policlinico Umberto I Hospital of 'Sapienza', University of Rome and the WCMC.

The blood flow dynamics in the thoracic aorta was assessed using flow-sensitive 4D Flow sequences acquired in the obliquesagittal orientation to encompass the entire thoracic aorta with prospective electrocardiography gating during free breathing, using a respiratory navigator and acquired in 20 phases/cardiac cycle (Fig. 1A). Sequence parameters were as follows: voxel sizing = $1.48 \times 1.48 \times 1.8 \mathrm{~mm}^{3}$, flip angle $=10-14^{\circ}$, echo time $=2.02-$ $2.15 \mathrm{~ms}$ and repetition time $=4.3-5.27 \mathrm{~ms}$; velocity encoding limits had been tailored (VENC $\sim 150 \mathrm{~cm} / \mathrm{s}$ ) as the lowest value avoiding aliasing (3dcine_ktarc_WIP package).

\section{CineMR imaging}

A stack of 4 contiguous 2D steady-state free-precession cine images (cineMR) covering the aortic root was acquired to analyse valve function, measure aortic root diameters and support the anatomical delimitation of aortic contours. Diameters of the ascending aorta were measured through additional cineMR images transverse to aortic long axis, next to the right pulmonary artery. The cineMR was also used to measure cardiac structure and function, left ventricle chamber volumes and ejection fraction, which were quantified based on planimetry of contiguous end-diastolic and end-systolic short-axis slices. Typical cineMR sequence parameters were as follows: 20 phases, slice thickness $6 \mathrm{~mm}$ for the aortic root/ $8 \mathrm{~mm}$ for the left ventricle, no overlap and no gap for the aortic root/gap: $2 \mathrm{~mm}$ for the left ventricle, reconstruction matrix $256 \times 256$, repetition time $3.58-3.97 \mathrm{~ms}$, echo time $1.42-1.60 \mathrm{~ms}$, flip angle $45-60^{\circ}$, in-plane resolution ranging between $0.73 \times 0.73 \mathrm{~mm}$ and $1.40 \times 1.40 \mathrm{~mm}$ (Fig. 1B).

Image analysis was performed using Cvi42 (v.5.3, Circle Cardiovascular Imaging, Calgary, Canada). Transverse diameters of the aortic root were measured in a standardized fashion based on the cusp commissure distance [20].

\section{D Flow data processing}

4D Flow data were visually inspected, and the time frame with the highest velocity-to-noise ratio was identified as the most representative of peak systole. At the peak systole, all images were processed through a semiautomated pipeline implemented through ad hoc in-house MATLAB ${ }^{\circledR}$ scripts (The MathWorks Inc., Natick, MA, USA):

1. CineMR and 4D Flow sequences were rigidly coregistered, and misalignments, if any, were manually corrected.

2. On cineMR images, the silhouette of the graft was manually traced. Also, the aortic-valve-free margin and the coronary ostia were traced on the 2 planes best allowing for their visualization (Fig. 1C).

3. 4D Flow data were automatically processed through prefiltering of velocity components to correct for eddy current errors and aliasing and to decrease $\operatorname{MR}$ noise $[16,21]$. The wall of the aortic lumen was manually traced on 4D Flow images to define the region of interest (ROI) on each slice of the acquired volume, exploiting the information obtained at Step 2 (Fig. 1D).

4. A $3 \mathrm{D}$ binary $\mathrm{RO}$ was defined as the cloud of voxels included in the closed isosurface encompassing the traced borders of the ROI [16]. This surface was smoothed through the Laplacian diffusion filtering to obtain a regularized surface mesh; the centre line of the vessel was created through skeletonization of the ROI. 
A

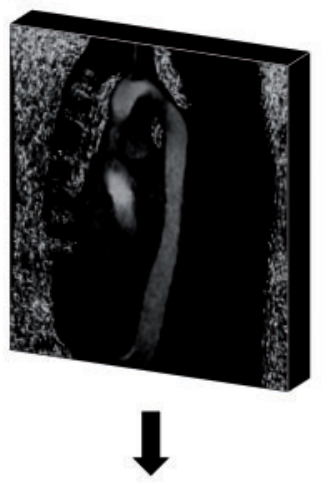

C

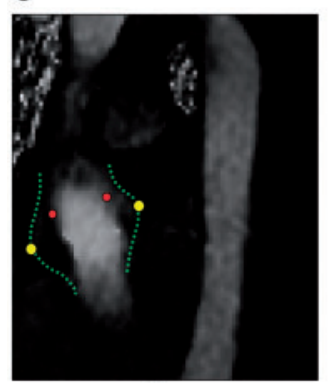

E

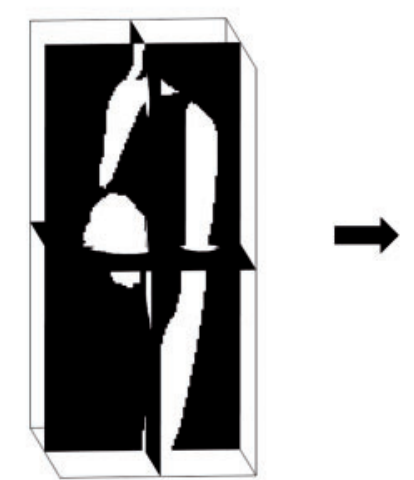

B

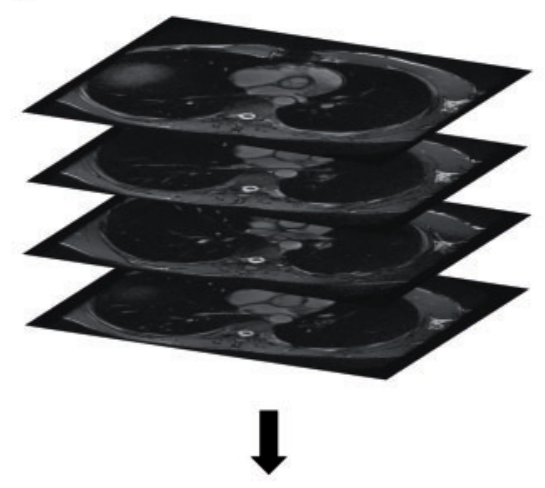

D
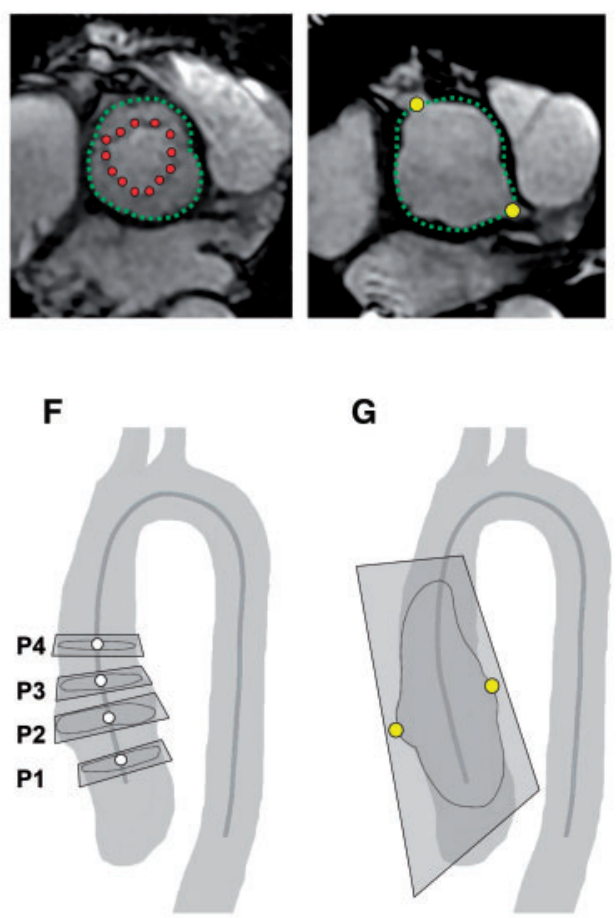

G

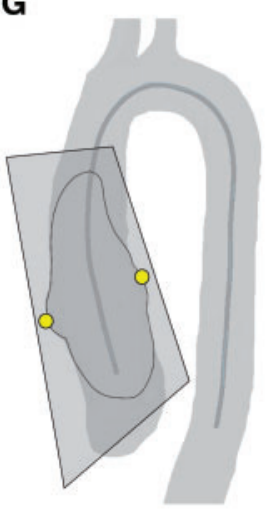

Figure 1: Pipeline of the 4D Flow analysis: (A) 4D Flow volume of acquisition oriented along an oblique-sagittal plane to encompass the entire thoracic aorta; (B) additional stack of 2D cineMR short-axis images covering the graft portion corresponding to the native aortic root; (C) tracing of the graft silhouette (green points) on cineMR images, including the aortic-valve-free margin (red points) and the coronary ostia (yellow points); (D) definition of the region of interest through manual tracing of the aortic lumen on each image of the 4D Flow sequence; (E) 3D binary region of interest consisting of all the voxels included in the closed iso-surface encompassing the traced borders of the aortic lumen; (F) definition of 4 cross-sections within the aortic root (P1: aortic annulus, P2: mid-portion of the aortic bulb, P3: sino-tubular junction, P4: cross-section $2 \mathrm{~cm}$ downstream of the sino-tubular junction) and (G) definition of a long-axis plane perpendicular to the cineMR planes and passing through the coronary ostia, for the computation of the associated in-plane streamlines.

5. According to previously published methods [16, 21], 4 crosssections (Fig. 1F) were manually defined: aortic annulus (P1), mid-portion of the aortic bulb (P2), sinotubular junction (P3) and the cross-section $2 \mathrm{~cm}$ downstream of the sino-tubular junction (P4). At P4, peak flow rate ( $\left.\mathrm{Q}_{\text {MAX }}\right)$, stroke volume and cardiac output were quantified. On each cross-section, velocity data were processed to compute the following: (i) peak and mean velocity magnitude $\left(|\mathrm{V}|_{\text {MAX }}\right.$ and $|\mathrm{V}|_{\text {mean, }}$, respectively); (ii) the space-dependent WSS vectors, assuming blood as a Newtonian fluid [16]. The mean value of WSS magnitudes $\left(|\overrightarrow{W S S}|_{\text {mean }}\right)$ and of WSS axial and circumferential components (WSS ax_mean $_{\text {and }}$ and WS $_{\text {cir_mean }}$ ) were extracted.
6. By means of the Paraview (ParaView, Sandia Corporation, Kitware Inc., NM, USA), a long-axis section of the ascending aorta was defined, corresponding to the plane perpendicular to the cineMR planes and passing through the coronary ostia (Fig. 1G). We computed the projection on this plane of the $3 \mathrm{D}$ velocity vectors whose origin was less than half voxel away from this plane. Projected vectors were used to compute the associated in-plane streamlines, i.e. the curves that are instantaneously tangent to the velocity vectors and describe the instantaneous trajectory of mass-less particles within the considered plane. Computed streamlines were clustered and colour coded according to the amount of 
Table 1: Population characteristics and cardiovascular magnetic resonance features

\begin{tabular}{|c|c|c|c|c|}
\hline & $\begin{array}{l}\text { WCMC group } \\
\text { straight tube graft } \\
(n=5)\end{array}$ & $\begin{array}{l}\text { EHR group graft } \\
\text { with neo-SV } \\
(n=5)\end{array}$ & $\begin{array}{l}\text { Overall } \\
(n=10)\end{array}$ & $P$-value \\
\hline Age (years), mean $\pm S D$ & $52.8 \pm 15.6$ & $51.2 \pm 11.1$ & $52 \pm 12.8$ & 1.000 \\
\hline Male gender, $n(\%)$ & $4(80)$ & $5(100)$ & $9(90)$ & \\
\hline $\mathrm{BSA}$, mean $\pm \mathrm{SD}$ & $1.95 \pm 0.28$ & $2.05 \pm 0.10$ & $2.00 \pm 0.2$ & 0.310 \\
\hline Time from operation (months), mean \pm SD & $29.9 \pm 10.2$ & $63 \pm 81.5$ & $46.5 \pm 57.5$ & 0.690 \\
\hline \multicolumn{5}{|l|}{ Aortic diameters } \\
\hline Diameter of prosthesis $(32 \mathrm{~mm} / 30 \mathrm{~mm} / 28 \mathrm{~mm}), n$ & $0 / 4 / 1$ & $1 / 3 / 1$ & & \\
\hline Annulus diameter $(\mathrm{cm})$, mean $\pm \mathrm{SD}$ & $26.5 \pm 1.3$ & $27.6 \pm 1.8$ & $27.1 \pm 1.6$ & 0.222 \\
\hline Root diameter $(\mathrm{cm})$, mean $\pm \mathrm{SD}$ & $27.6 \pm 1.1$ & $35 \pm 1.6$ & $31.3 \pm 4.0$ & $0.008^{*}$ \\
\hline Ascending aortic diameter ${ }^{\mathrm{a}}(\mathrm{cm})$, mean $\pm \mathrm{SD}$ & $29 \pm 1.7$ & $30.9 \pm 2.3$ & $30.0 \pm 2.2$ & 0.310 \\
\hline Aortic arch diameter $(\mathrm{cm})$, mean $\pm \mathrm{SD}$ & $26 \pm 3.8$ & $27.2 \pm 6.4$ & $26.6 \pm 5.1$ & 1.000 \\
\hline Descending aortic diameter $(\mathrm{cm})$, mean $\pm \mathrm{SD}$ & $24.7 \pm 2.1$ & $23.6 \pm 4.4$ & $24.2 \pm 3.4$ & 0.690 \\
\hline \multicolumn{5}{|l|}{ Left ventricular function, mean $\pm S D$} \\
\hline LV end-diastolic volume $\left(\mathrm{ml} / \mathrm{m}^{2}\right)$ & $77.6 \pm 12.5$ & $79.1 \pm 14.2$ & $78.3 \pm 12.6$ & 1.000 \\
\hline LV end-systolic volume $\left(\mathrm{ml} / \mathrm{m}^{2}\right)$ & $30.5 \pm 9.3$ & $33.4 \pm 6.1$ & $31.9 \pm 7.5$ & 0.690 \\
\hline LV stroke volume $\left(\mathrm{ml} / \mathrm{m}^{2}\right)$ & $47.1 \pm 4.5$ & $45.6 \pm 9.8$ & $46.4 \pm 7.3$ & 0.841 \\
\hline Cardiac output (I/min) & $5.5 \pm 1.7$ & $5.0 \pm 0.9$ & $5.3 \pm 1.3$ & 0.841 \\
\hline LV ejection fraction (\%) & $63.1 \pm 6.0$ & $57.2 \pm 4.5$ & $60.1 \pm 5.9$ & 0.056 \\
\hline $\mathrm{LV}$ mass $\left(\mathrm{g} / \mathrm{m}^{2}\right)$ & $66.6 \pm 9.1$ & $57.1 \pm 7.1$ & $61.8 \pm 9.2$ & 0.151 \\
\hline
\end{tabular}

rotational flow, i.e. the angle spanned by the in-plane velocity vectors associated with each streamline.

\section{Statistical analysis}

Continuous variables were expressed in terms of mean \pm standard deviation (aortic dimensions and left ventricular volumes) or minimum-to-maximum range (extracted 4D Flow variables) and compared using the non-parametric Mann-Whitney test. Differences with $P<0.05$ were considered statistically significant.

\section{RESULTS}

Diagnostic 4D Flow and cineMR datasets were successfully acquired in all patients with no complications. No significant differences were noted in heart rate values between the straight tube graft and graft with neo-SV groups during 4D Flow sequence acquisition (mean \pm standard deviation: $60.6 \pm 6.2 \mathrm{bpm}$ vs $59.6 \pm 9.4 \mathrm{bpm}$, respectively, $P=0.898$ ). Table 1 lists the characteristics of the study participants and cineMR-based anatomical and functional measurements. The comparison of aortic diameters revealed a larger aortic root size in the group with neo-SV graft $(P=0.008)$.

\section{D Flow-based fluid dynamics analysis}

Bulk flow. No significant differences were noted in $Q_{\text {MAX, }}$ stroke volume and cardiac output between 2 groups $(20.3 \div 29.3 \mathrm{l} / \mathrm{min}$, $54.7 \div 97.2 \mathrm{ml}$ and $3.8 \div 5.0 \mathrm{l} / \mathrm{min}$ in the graft with neo-SV group and $19.7 \div 31.2 \mathrm{l} / \mathrm{min}, 59.6 \div 100.7 \mathrm{ml}$ and $3.8 \div 5.7 \mathrm{l} / \mathrm{min}$ in the straight tube graft group, respectively; $P$ : 0.84-1.00). At peak systole, a centred and aligned aortic flow was observed in both groups. Over the 4 analysed cross-sections, $|\mathrm{V}|_{\text {MAX }}$ ranged from 90.0 to $180.1 \mathrm{~cm} / \mathrm{s}$ in the neo-SV group and from 75.3 to $192.2 \mathrm{~cm} / \mathrm{s}$ in the straight tube group, and at no cross-section level, statistically significant differences were detected (Table 2). Instead, a trend towards higher $|\mathrm{V}|_{\text {mean }}$ values was observed in the straight tube group when compared with the neosinuses group, with a significant difference at Section P1 $(P=0.0317)$ and close to statistically significant at Section P2 $(P=0.0952)$.

This result was consistent with the differences in velocity profiles at the proximal aortic root: in the neo-SV group, peripheral slow-flow regions with velocity magnitudes largely below $50 \mathrm{~cm}$ / $s$ were systematically visible within the neosinuses (Fig. 2a), whereas in the straight tube group, these regions were minimal and localized close to the aortic wall (Fig. 2b).

Wall shear stress. In the neo-SV group, $|\overrightarrow{W S S}|_{\text {mean }}$ was lower compared with those computed for the straight tube group at Sections P1-P3, and differences were almost statistically significant at Section P1 $(P=0.0952)$ and statistically significant at Sections P2-P3 $(P=0.0159$ and $P=0.0079$, respectively). These differences were almost exclusively due to the WSS axial component (Table 2).

Rotational flows. Organized vortical flows at the SV level were observed only in the neo-SV group (Fig. $3 A$ ). In the left $(L)$ and the right $(\mathrm{R})$ coronary neo-SV, the local analysis of velocity vectors highlighted a common rotation pattern, with blood progressively moving from the aortic central jet towards the wall and rotating backwards within each neo-SV (Fig. 3B) by an angle $\phi$ $>270^{\circ}$ in the majority of the neo-SV volume (Fig. 3C). The analysis of the frequency distribution of the in-plane streamlines based on the respective amount of rotation confirmed this result (Fig. 4). Even when considering the whole domain, in the neo-SV 
Table 2: Results of 4D Flow analysis on the 4 selected crosssections (P1-P4) encompassing the entire aortic root

\begin{tabular}{|c|c|c|c|}
\hline Planes & Neosinuses & Straight tube & $P$-value \\
\hline \multicolumn{4}{|c|}{$|\mathrm{V}|_{\text {MAX }}(\mathrm{cm} / \mathrm{s})$} \\
\hline P1 & $100.6 \div 170.7$ & $117.5 \div 192.2$ & 0.3095 \\
\hline P2 & $116.4 \div 180.1$ & $89.8 \div 177.0$ & 0.4206 \\
\hline P3 & $95.3 \div 155.2$ & $94.8 \div 159.1$ & 0.5476 \\
\hline P4 & $90.0 \div 123.7$ & $75.3 \div 139.8$ & 0.4206 \\
\hline \multicolumn{4}{|c|}{$|\mathrm{V}|_{\text {mean }}(\mathrm{cm} / \mathrm{s})$} \\
\hline P1 & $42.0 \div 58.7$ & $55.6 \div 83.4$ & 0.0317 \\
\hline P2 & $37.9 \div 60.9$ & $50.9 \div 89.9$ & 0.0952 \\
\hline P3 & $38.8 \div 62.6$ & $44.6 \div 75.1$ & 0.2222 \\
\hline P4 & $41.0 \div 67.2$ & $43.4 \div 60.2$ & 0.5476 \\
\hline \multicolumn{4}{|c|}{$|\overrightarrow{\mathrm{WSS}}|_{\text {mean }}(\mathrm{Pa})$} \\
\hline P1 & $0.11 \div 0.25$ & $0.15 \div 0.41$ & 0.0952 \\
\hline P2 & $0.11 \div 0.29$ & $0.23 \div 0.43$ & 0.0159 \\
\hline P3 & $0.17 \div 0.25$ & $0.27 \div 0.56$ & 0.0079 \\
\hline P4 & $0.12 \div 0.69$ & $0.24 \div 0.53$ & 0.4206 \\
\hline \multicolumn{4}{|c|}{$W S S_{\text {ax_mean }}(\mathrm{Pa})$} \\
\hline P1 & $0.07 \div 0.18$ & $0.10 \div 0.39$ & 0.0952 \\
\hline P2 & $0.05 \div 0.25$ & $0.19 \div 0.41$ & 0.0159 \\
\hline P3 & $0.11 \div 0.21$ & $0.22 \div 0.52$ & 0.0079 \\
\hline P4 & $0.08 \div 0.60$ & $0.22 \div 0.47$ & 0.4206 \\
\hline \multicolumn{4}{|c|}{$W_{S S_{\text {circ_mean }}(\mathrm{Pa})}$} \\
\hline P1 & $0.07 \div 0.13$ & $0.09 \div 0.17$ & 0.2222 \\
\hline P2 & $0.06 \div 0.15$ & $0.07 \div 0.14$ & 1.0 \\
\hline P3 & $0.09 \div 0.11$ & $0.05 \div 0.18$ & 0.5476 \\
\hline P4 & $0.07 \div 0.27$ & $0.05 \div 0.17$ & 0.8413 \\
\hline
\end{tabular}

Data are expressed as $\min \div \max$ values. The non-parametric MannWhitney test $(P<0.05)$. For each variable, the range between the minimum value and the maximum value computed on the single plane is reported. $|\mathrm{V}|_{\text {MAx: }}$ peak velocity magnitude; $|\mathrm{V}|_{\text {mean }}$ : mean velocity magnitude; $|\overrightarrow{W S S}|_{\text {mean }}$ : mean WSS magnitude; $\overrightarrow{W S S}_{a x}$ : mean WSS axial component; $\overrightarrow{W S S}_{\text {circ }}$ : mean WSS circumferential component. group, more than $10 \%$ of the in-plane streamlines had a high degree of rotation $\left(\phi>270^{\circ}\right)$, this amount being almost 3-times higher $(P=0.008)$ than in the straight tube graft $(2.2 \div 5.7 \%$ vs $10.3 \div 14.0 \%)$. The amount of streamlines with a low degree of rotation $\left(0^{\circ}<<90^{\circ}\right)$ was approximately $10 \%$ higher in the straight tube graft group (range $=92.7 \div 96.0 \%$ ) when compared with the graft with the neosinuses group (range $=82.5 \div 89.5 \%$ ).

\section{DISCUSSION}

This study provides several key findings regarding the impact of surgical technique on aortic root flow physiology. First, straight tube VSRR yielded higher mean aortic flow velocity at the aortic annulus when compared with neosinus reconstruction $(P=0.03)$ with no significant differences in peak velocity. Second, differences in the aortic flow were paralleled by increased WSS in the axial plane at the proximal aortic root. Third, in the prosthesis with neo-SV, there is a clear distinction between fast central laminar flow $(82.5 \div 89.5 \%$ of streamlines) and slow peripheral turbulent flow with vortex generation $(10.3 \div 14.0 \%)$, whereas this is not the case in the straight tube graft. Taken together, our results support the notion that aortic blood flow pattern is not solely dependent on intact aortic valve opening but is also affected by aortic root geometry.

Previously, Oechtering et al. [22] demonstrated similar SV vortices in patients with an anatomically shaped sinus prosthesis and normal controls, even though a tendency towards more pronounced SV vortex formation was observed in surgical patients as assessed by visual grading of vortex size and maximal retrograde velocity.

Another preliminary investigation in a patient with the same prosthesis did not detect any vortex in the neosinuses, but the method used had major limitations in terms of spatial resolution [23].

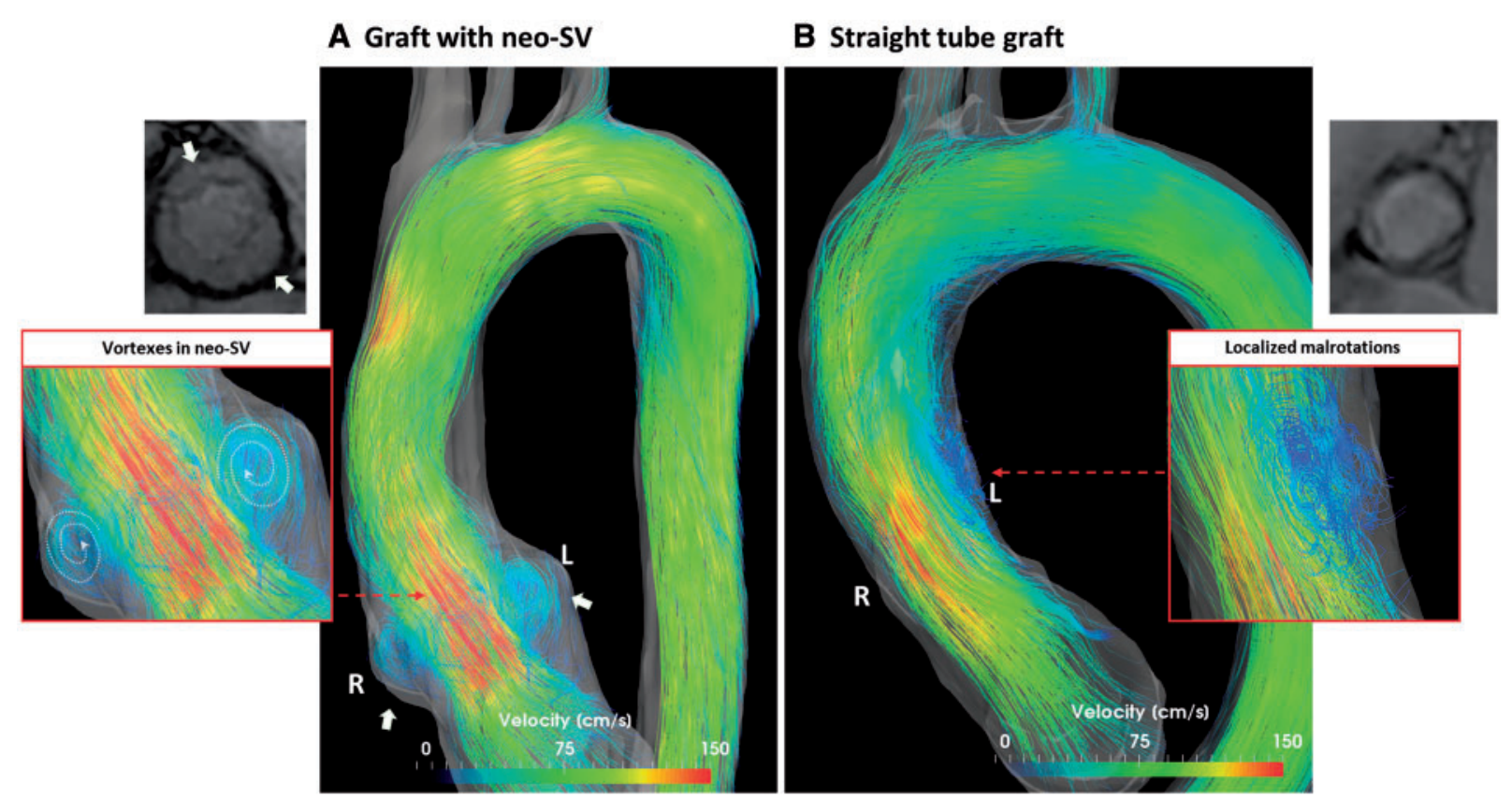

Figure 2: 3D streamlines describing the bulk flow field at peak systole for 2 patients implanted with a graft with neo-SV (A) and a straight tube graft (B), respectively. Streamlines are colour coded based on the instantaneous and local 3D velocity magnitude. For each panel in the upper small box, the cineMR corresponding to the plane passing through the aortic root is represented, and the lower boxes show the vortices of the neo-SV graft (left) and the disorganized flow within straight tubular graft (right). 


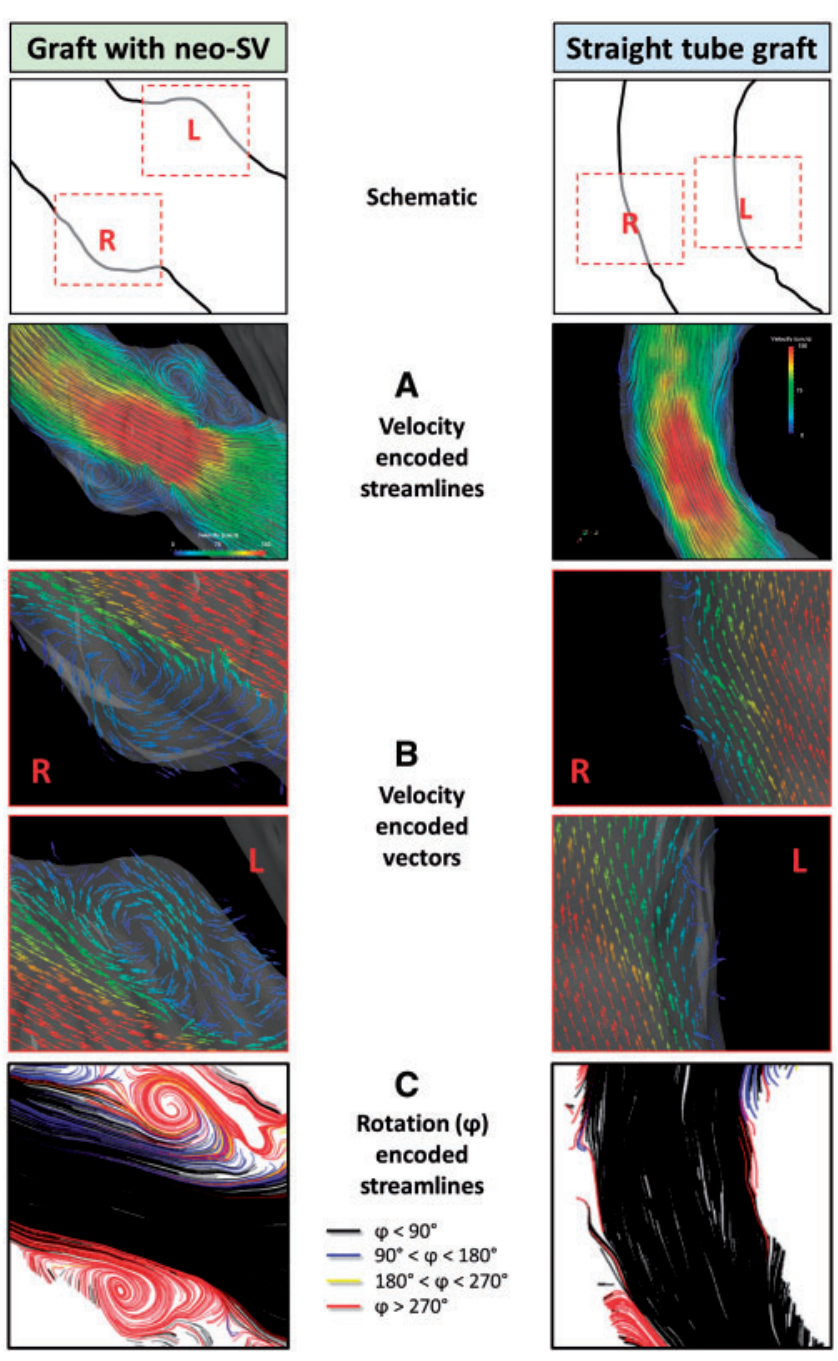

Figure 3: Analysis of in-plane streamlines on the long-axis section of the ascending aorta passing through the coronary ostia, for 2 patients implanted with a neosinuses graft (left panel) and a straight tube graft (right panel), respectively. For each patient, the following information is depicted (from top to bottom panels): sketch of the portion of the ascending aorta relevant to this analysis, depicting regions of the graft corresponding to the location of the native left and right sinuses ( $L$ and $R$ ); in-plane streamlines colour coded based on the instantaneous and local 3D velocity magnitude $(\mathbf{A})$; zoomed view of regions $L$ and $R$, where velocity vectors are depicted to highlight the direction of the streamlines (B) and in-plane streamlines colour coded based on the respective amount of rotation, i.e. the angle spanned by each streamline $(\mathbf{C})$.

Markl et al. [24] reported that, although vortex formation was enhanced by the re-creation of neo-SV, normal vorticity was visually detected even in the patients with straight tube graft.

Our study is the first quantitative demonstration of a greater amount and organization of vortical flows in the patients with neo-SV when compared with the patients with straight tube prostheses.

This discordance could be due to the preliminary delineation of the graft margins on the cineMR images prior to processing 4D Flow data used in our study, which could ensure that we avoided misinterpreting false rotating-streamlines near the graft due to background noise or other nearby vessel flows.

Our findings in patients with straight tube grafts are similar to those of Oechtering et al. [25] who reported the presence of small systolic vortices in the neoroot with a rotation axis orthogonal to the prosthesis wall, which was angled approximately $90^{\circ}$ along the vessel axis ('malrotated' vortices).

In our series, the turbulent flow generated in the straight tube is inconstant, disorganized and asymmetric and did not seem to respect any rotational axis, as opposed to the grafts with neo-SV (Fig. 2).

We can hypothesize that this disorganized turbulent flow dissipates the response energy to the pulse wave exerted by the Dacron wall in patients with straight tube grafts. The recirculation zone offered by the neo-SV might warrant a reservoir effect that canalized the response energy in organized vortices.

The analysis of velocity streamlines according to the degree of rotation revealed a gradual transition from the vectors of laminar flow at the centre of the bloodstream to regions at a high degree of rotation $\left(\phi>270^{\circ}\right)$ at the border of the root graft, which is significantly more pronounced in patients with reconstruction of the neo-SV (Figs 3 and 4). This could result in a better valve mechanism, as demonstrated in a simulation model using fluidstructure interaction finite-element analysis [7].

Replacement of a portion of the ascending aorta with the Dacron material in VSRR leads to reduced compliance due to the absence of the normal physiological Windkessel effect. This results in an increase in valve peak velocities and aortic flow acceleration [26]. We did not find any significant differences in aortic peak velocity values between the 2 surgical approaches. Conversely, mean velocity was significantly reduced in patients with neo-SV at the aortic annulus, and a trend (not statistically significant) towards lower mean flow velocity values is appreciable in the graft with the neo-SV group at all other levels. Although the lower mean velocity at the aortic bulb in the neoSV graft can be presumably due to the larger mean diameter and recirculation in the low-velocity regions of the neo-SV, this difference at the aortic annulus and other levels requires further extensive investigation with a larger population. Indeed, it is not possible to exclude that this discrepancy in mean velocity values is due to the small non-significant differences in graft diameters, cardiac output and stroke volume.

A significant reduction in mean WSS magnitude and WSS axial component was found at the bulb and sino-tubular junction levels in patients with neo-SV reconstruction when compared with the patients with straight tube grafts $(P=0.007$ and 0.015 , respectively). A better leaflet-sinus assembly in patients with neosinuses probably explains the minor tension at the annulus. In fact, in another finite-element simulation, Beck et al. [27] tested aortic grafts under pressure and found a major stress concentration along the leaflet attachment in straight tube grafts. Conversely, in the root graft where the neo-SV expanded in a uniform manner, a proper leaflet-sinus assembly was maintained, and the leaflet stress was reduced. The complexity of these dynamic phenomena might confer a lower rigidity of the aortic root-valve system in patients with neo-SV; the consequent more homogeneous energy propagation might reduce the working stress on the valve and potentially slow down the structural deterioration of the leaflet. Furthermore, a decreased WSS at the bulb implicates decreased tension on the coronary buttons that might potentially reduce the incidence of bleeding or late pseudoaneurysm formation [28].

In our experience, vortical flow persists for a long time in the graft with neosinuses, even during early diastole (Supplementary Material, Fig. S1), confirming previous studies [22]. This could suggest a key role of these rotational streams in maintaining kinetic energy, which is of crucial importance in a more 


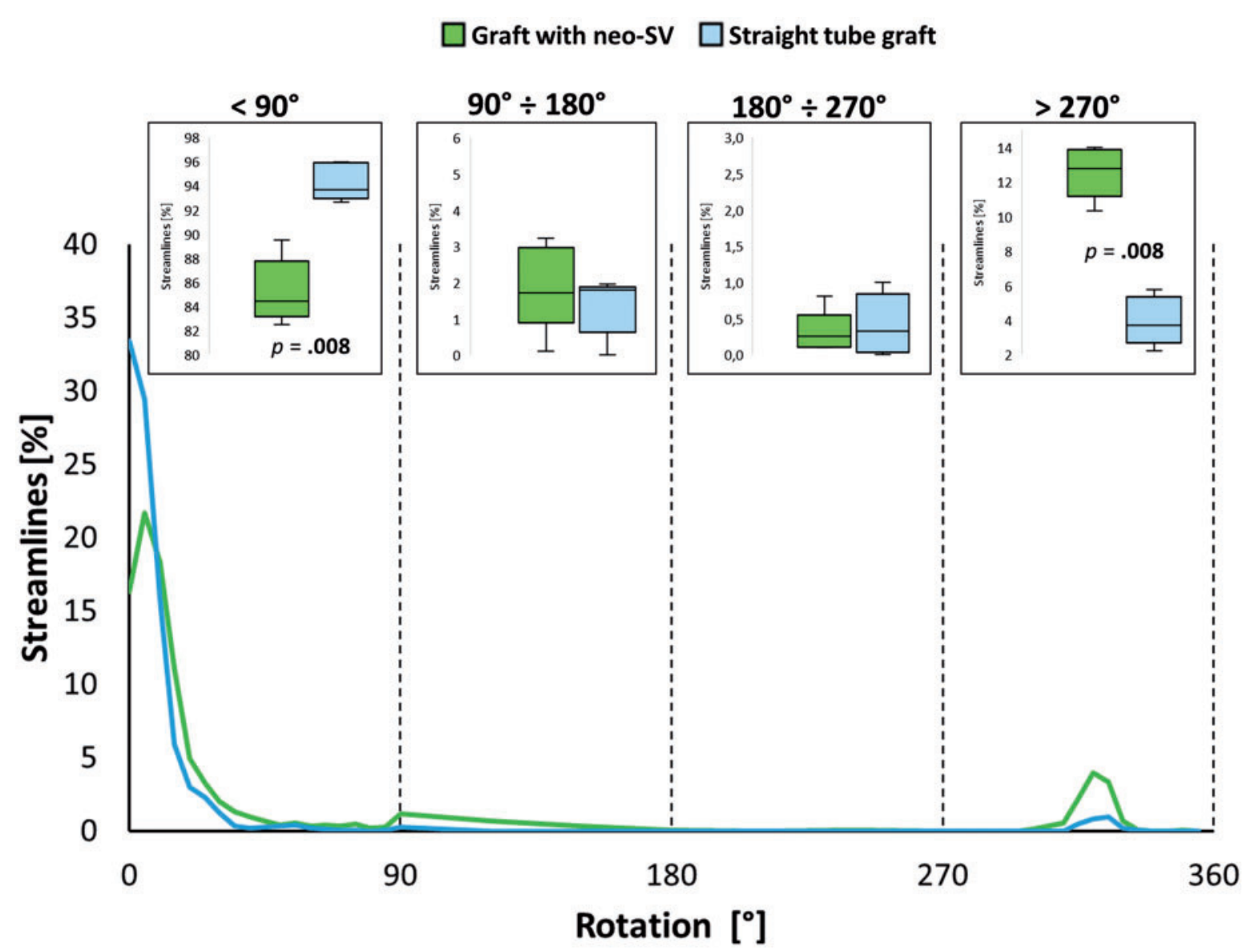

Figure 4: Frequency distribution of the in-plane streamlines according to the respective amount of rotation, i.e. the angle spanned by each streamline. Data were obtained for every patient of each group (green = neosinuses graft patients, light blue = straight tube graft patients). Continuous lines represent the median value over each group of patients. Box plots represent the distribution of data as observed when clustering the streamlines based on the rotation angle: boxes range from the 25 th to the 75 th percentile, and the median value is indicated. Whiskers indicate the minimum and maximum value.

continuous and homogeneous supply of coronary blood flow during the entire cycle. Moreover, the clock-wise-rotating vortexes occurring in the neosinuses at the end of systole might aid the closure of the valve leaflets: vortex forming in the SV at the late systole and persistent in early diastole could be determinant in regulating smooth and progressive aortic valve closure, ensuring a synchronous, homogeneous and stress-free closure [7] Further studies, through MR systems with higher spatial and temporal resolution, will enable to explore the key role of the SV and vortex in the modulation of coronary blood flow and in the regulation of valve leaflets biomechanics.

From a clinical point of view, it must be mentioned that there are several studies reporting on good long-term results in patients with straight tube grafts [29] and prostheses with neo-SV [10-12]. Although modified prostheses have been increasingly used across the world, the number of reports on the long-term results is still low. In a recent study [11], re-creation of the SV did not affect short-term clinical outcomes and aortic valve status when compared with straight tube grafts. On the other hand, the series with the longest follow-up to date (13 years) reported survival rate and freedom from residual aortic insufficiency comparable with the majority of long-term follow-up studies using straight tube grafts [10]. Because of the low incidence of events in VSRR patients, a larger sample size and longer follow-up are certainly needed to evaluate eventual differences in clinical outcomes. However, as VSRR procedures are often performed in young and active patients, it is important to establish the technical variations that assure a more physiological aortic root flow even in stress conditions.

This study has several limitations. The sample size is extremely limited, and therefore our results must be viewed as exploratory and need confirmation on a larger number of patients. Also, we did not have a group of healthy subjects as controls, and so, even though the flow in the neo-SV groups visually resembles that of the normal aorta, we cannot objectively conclude that recreation of the SV leads to more physiological dynamics.

Finally, on a technical note, in this type of surgery, the reimplantation of the aortic leaflets, with re-establishment of an effective leaflet coaptation and absence of tension and distortion of the commissural posts, is likely more important than SV reconstruction. It is highly likely that a technically imperfect valve reimplantation would affect valve function much more than recreation of the neo-SV.

\section{CONCLUSION}

In conclusion, these preliminary data suggest that neo-SV reconstruction during VSRR is associated with a significantly lower WSS and organized vortical flows at the level of the sinus that are not 
evident using the straight tube graft. If these results can be confirmed in larger studies, it is possible that neo-SV reconstruction provides advantages in terms of aortic valve function and durability after VSRR.

\section{SUPPLEMENTARY MATERIAL}

Supplementary material is available at ICVTS online.

\section{ACKNOWLEDGEMENTS}

IRCCS Policlinico San Donato is a Clinical Research Hospital partially funded by the Italian Ministry of Health.

Conflict of interest: As inventor of the Valsalva graft described in the manuscript, Dr De Paulis receives royalties from Vascutek Terumo. All other authors have nothing to disclose.

\section{REFERENCES}

[1] Charitos El, Stierle U, Tietze C, Hanke T, Karluss A, Misfeld M et al. Clinical outcomes and lessons learned with aortic valve repair in 508 patients. J Heart Valve Dis 2014;23:550-7.

[2] David TE, Feindel CM. An aortic valve-sparing operation for patients with aortic incompetence and aneurysm of the ascending aorta. J Thorac Cardiovasc Surg 1992;103:617-21.

[3] Gaudino M, Weltert L, Munjal M, Lau C, Elsayed M, Salica A et al. Early clinical outcome after aortic root replacement using a biological composite valved graft with and without neo-sinuses. Eur J Cardiothorac Surg 2017;51:316-21

[4] De Paulis R, De Matteis GM, Nardi P, Scaffa R, Buratta MM, Chiariello L. Opening and closing characteristics of the aortic valve after valvesparing procedures using a new aortic root conduit. Ann Thorac Surg 2001;72:487-94

[5] Pisani G, Scaffa R, leropoli O, Dell'Amico EM, Maselli D, Morbiducci U et al. Role of the sinuses of Valsalva on the opening of the aortic valve. J Thorac Cardiovasc Surg 2013;145:999-1003.

[6] Peacock JA. An in vitro study of the onset of turbulence in the sinus of Valsalva. Circ Res 1990;67:448-60.

[7] Katayama S, Umetani N, Sugiura S, Hisada T. The sinus of Valsalva relieves abnormal stress on aortic valve leaflets by facilitating smooth closure. J Thorac Cardiovasc Surg 2008;136:1528-35.

[8] Ohta Y, Kikuta Y, Shimooka T, Mitamura Y, Yuhta T, Dohi T. Effect of the sinus of Valsalva on the closing motion of bileaflet prosthetic heart valves. Artif Organs 2000;24:309-12

[9] Girardi LN, Gaudino MF. Valve-sparing root replacement: still so much to learn. J Thorac Cardiovasc Surg 2017;154:798-9.

[10] De Paulis R, Chirichilli I, Scaffa R, Weltert L, Maselli D, Salica A et al. Long-term results of the valve reimplantation technique using a graft with sinuses. J Thorac Cardiovasc Surg 2016;151:112-9.

[11] Gaudino M, Di Franco A, Weltert L, Benedetto U, Lau C, Gambardella et al. The role of neo-sinus reconstruction in aortic valve-sparing surgery. J Card Surg 2017;32:328-33.

[12] Gaudino M, Di Franco A, Ohmes LB, Weltert L, Lau C, Gambardella I et al. Biological solutions to aortic root replacement: valve-sparing versus bioprosthetic conduit. Interact CardioVasc Thorac Surg 2017;24: 855-61.

[13] Markl M, Schnell S, Wu C, Bollache E, Jarvis K, Barker AJ et al. Advanced flow MRI: emerging techniques and applications. Clin Radiol 2016;71 779-95.
[14] Stankovic Z, Allen BD, Garcia J, Jarvis KB, Markl M. 4D Flow imaging with MRI. Cardiovasc Diagn Ther 2014;4:173-92.

[15] Potters WV, Marquering HA, VanBavel E, Nederveen AJ. Measuring wall shear stress using velocity-encoded MRI. Curr Cardiovasc Imaging Rep 2014;7:9257.

[16] Piatti F, Sturla F, Bissell MM, Pirola S, Lombardi M, Nesteruk I et al. 4D Flow analysis of BAV-related fluid-dynamic alterations: evidences of wall shear stress alterations in absence of clinically-relevant aortic anatomical remodeling. Front Physiol 2017;8:441.

[17] Erbel R, Aboyans V, Boileau C, Bossone E, Bartolomeo RD, Eggebrecht $H$ et al. 2014 ESC guidelines on the diagnosis and treatment of aortic diseases: document covering acute and chronic aortic diseases of the thoracic and abdominal aorta of the adult. The task force for the diagnosis and treatment of aortic diseases of the European Society of Cardiology (ESC). Eur Heart J 2014;35:2873-926.

[18] Hiratzka LF, Bakris GL, Beckman JA, Bersin RM, Carr VF, Casey DE Jr et al. 2010 ACCF/AHA/AATS/ACR/ASA/SCA/SCAI/SIR/STS/SVM guidelines for the diagnosis and management of patients with thoracic aortic disease: a report of the American College of Cardiology Foundation/American Heart Association Task Force on Practice Guidelines, American Association for Thoracic Surgery, American College of Radiology, American Stroke Association, Society of Cardiovascular Anesthesiologists, Society for Cardiovascular Angiography and Interventions, Society of Interventional Radiology, Society of Thoracic Surgeons, and Society for Vascular Medicine. Circulation 2010;121:e266-369.

[19] Gaudino M, Lau C, Munjal M, Avgerinos D, Girardi LN. Contemporary outcomes of surgery for aortic root aneurysms: a propensity-matched comparison of valve-sparing and composite valve graft replacement. J Thorac Cardiovasc Surg 2015;150:1120-9.

[20] Burman ED, Keegan J, Kilner PJ. Aortic root measurement by cardiovascular magnetic resonance: specification of planes and lines of measurement and corresponding normal values. Circ Cardiovasc Imaging 2008; 1:104-13.

[21] Piatti F, Pirola S, Bissell M, Nesteruk I, Sturla F, Della Corte A et al. Towards the improved quantification of in vivo abnormal wall shear stresses in BAV-affected patients from 4D-flow imaging: benchmarking and application to real data. J Biomech 2017;50:93-101.

[22] Oechtering $T H$, Hons $C F$, Sieren $M$, Hunold $P$, Hennemuth $A$, Huellebrand $M$ et al. Time-resolved 3-dimensional magnetic resonance phase contrast imaging (4D Flow MRI) analysis of hemodynamics in valve-sparing aortic root repair with an anatomically shaped sinus prosthesis. J Thorac Cardiovasc Surg 2016;152:418-27.

[23] Frydrychowicz A, Berger A, Stalder AF, Markl M. Preliminary results by flow-sensitive magnetic resonance imaging after Tiron David I procedure with an anatomically shaped ascending aortic graft. Interact CardioVasc Thorac Surg 2009;9:155-8.

[24] Markl M, Draney MT, Miller DC, Levin JM, Williamson EE, Pelc NJ et al. Time-resolved three-dimensional magnetic resonance velocity mapping of aortic flow in healthy volunteers and patients after valve-sparing aortic root replacement. J Thorac Cardiovasc Surg 2005;130:456-63.

[25] Oechtering $\mathrm{TH}$, Frydrychowicz A, Sievers $\mathrm{HH}$. Malrotated sinus vortices in straight graft valve-sparing aortic root treatment: a matter of concern? J Thorac Cardiovasc Surg 2017; 154:794-7.

[26] Semaan E, Markl M, Malaisrie SC, Barker A, Allen B, McCarthy P et al. Haemodynamic outcome at four-dimensional flow magnetic resonance imaging following valve-sparing aortic root replacement with tricuspid and bicuspid valve morphology. Eur J Cardiothorac Surg 2014;45: 818-25.

[27] Beck A, Thubrikar MJ, Robicsek F. Stress analysis of the aortic valve with and without the sinuses of Valsalva. J Heart Valve Dis 2001;10:1-11.

[28] Weltert L, De Paulis R, Scaffa R, Maselli D, Bellisario A, D'Alessandro S. Re-creation of a sinuslike graft expansion in Bentall procedure reduces stress at the coronary button anastomoses: a finite element study. J Thorac Cardiovasc Surg 2009;137:1082-7.

[29] Beckmann E, Martens A, Pertz J, Kaufeld T, Umminger J, Hanke JS et al. Valve-sparing David I procedure in acute aortic type A dissection: a 20year experience with more than 100 patients. Eur J Cardiothorac Surg 2017;52:319-24. 